\title{
Bi-stage time evolution of nano-morphology on inductively coupled plasma etched fused silica surface caused by surface morphological transformation
}

\author{
Xiaolong Jiang ${ }^{a}{ }^{1}{ }^{1}$, Lijuan Zhang ${ }^{a}$, Yang Bai ${ }^{a}$, Ying Liu ${ }^{b} *^{2}$, Zhengkun Liu ${ }^{b}$, Keqiang Qiu ${ }^{b}$, \\ Wei Liao ${ }^{a}$, Chuanchao Zhang ${ }^{a}$, Ke Yang ${ }^{a}$, Jing Chen ${ }^{a}$, Yilan Jiang ${ }^{a}$, Xiaodong Yuan ${ }^{a}$ \\ ${ }^{a}$ Research Center of laser Fusion, China Academy of Engineering Physics, Mianyang 621900, \\ China \\ ${ }^{b}$ National Synchrotron Radiation Laboratory, University of Science and Technology of China, hefei \\ 230029, China \\ *1 Corresponding author: Email: Jxl0290@mail.ustc.edu.cn \\ *² Corresponding author: Email: liuychch@ustc.edu.cn
}

\begin{abstract}
In this work, we experimentally investigate the surface nano-roughness during the inductively coupled plasma etching of fused silica, and discover a novel bi-stage time evolution of surface nano-morphology. At the beginning, the rms roughness, correlation length and nano-mound dimensions increase linearly and rapidly with etching time. At the second stage, the roughening process slows down dramatically. The switch of evolution stage synchronizes with the morphological change from dual-scale roughness comprising long wavelength underlying surface and superimposed nano-mounds to one scale of nano-mounds. A theoretical model based on surface morphological change is proposed. The key idea is that at the beginning, etched surface is dual-scale, and both larger deposition rate of etch inhibitors and better plasma etching resistance at the surface peaks than surface valleys contribute to the roughness development. After surface morphology transforming into one-scale, the difference of plasma resistance between surface peaks and valleys vanishes, thus the roughening process slows down.
\end{abstract}

Keywords: inductively coupled plasma etching; Plasma etching; fused silica; roughness; Bi-stage evolution; morphology;

\section{Introduction}

In the field of optics, fused silica is an ideal material that has been widely used in ultraviolet and infrared optical components due to its high transmissivity, low coefficient of thermal expansion and excellent mechanical properties [1, 2]. During the fabrication of fused silica optics, plasma etching is a very important technique that has been routinely used in pattern transferring [3, 4], substrate finishing [5, 6] and anti-reflection surface nano-texturing $[7,8]$. Plasma etching can be categorized into at least three types: conventional plasma etching (neutral chemical reaction only) [9], reactive plasma etching (RIE) [6] and high density plasma etching [4]. In conventional plasma etching, the sample is grounded and the main etching mechanism is chemical etching by isotropic neutral radicals. In RIE, the sample is biased or floating, both isotropic neutral radicals and anisotropic ions contribute to the etching. For highdensity plasma etching, the ratio of ions to reactive neutrals is so large that ion etching is 
dominant. As a high density plasma etching, inductively couple plasma (ICP) etching is nowadays the most popular high plasma etching process [4, 10-12]. It combines plasma etching with ion bombardment at relatively low pressure, making the etching highly anisotropic. Moreover, unlike RIE, substrate power and coil power are independent of each other in ICP etching systems, thus excellent control over plasma density and ion bombardment energy is provided.

However, plasma etching often causes the development of roughness on etched surface [10, 13-21]. This influence could be detrimental or beneficial dependent on the application background. In pattern transfer and substrate finishing processes, surface roughening generally degrades the device performances. While, in anti-reflection surface nano-texturing, plasma induced sub-wavelength structures could act as a graded refractive index layer and reduce the interface reflection. Therefore, understanding the formation and development of plasma induced roughening on fused silica surfaces is of primary importance.

Most of the previous works on plasma induced roughness concentrated on conventional plasma [9] or RIE etching of silicon [17-19] or polymer films [20, 21]. However, roughness in high density plasma is different because reactive neutrals to ion flux impinging on the substrate plays an important role in the roughening [22]. As to the time evolution of roughness, the main founding is the linear or approximate linear increase of $\mathrm{rms}$ and correlation length $[9,17,18]$. In this work, we quantitatively investigate the formation, development and evolution mechanism of nano-mounds during the ICP etching of fused silica surface for the first time. The main contributions are the identification of a novel bi-stage roughness evolution and the theoretical model proposed to explain it. First, a morphological transformation is identified. At the beginning of the etching, etched surface exhibits dual-scale nano-morphology consisting of long wavelength underlying background surface and superimposed nano-mounds. After certain point, the nano-mounds, which grow with etching time, become so dominant that the underlying background vanishes. Consequently, surface nano-morphology transforms from dual-scale into one-scale. Then we studied the time evolutions of rms roughness, correlation length and nano-mound dimensions. And their time evolutions all comprise two linear subprocesses. These differ from the one-stage evolutions reported in previous works, and suggest a fundamental change of evolution mechanism. At last, a theoretical model based on surface morphological change is proposed. During the period of dual-scale morphology, both larger deposition rate of etch inhibitors and better plasma etching resistance at the surface peaks than surface valleys contribute to the surface roughening. After surface morphology transforms into one-scale, the plasma etching resistance of surface peaks drops to the level of valleys, and consequently the increases of mound height as well as surface roughness slow down.

\section{Experimental}

Fused silica samples (JGS1) were obtained from Sikoy Optics \&Electron Co. Ltd. with dimensions of $50 \mathrm{~mm} \times 50 \mathrm{~mm} \times 2 \mathrm{~mm}$. Each sample was optically polished with surface roughness of $0.56 \mathrm{~nm}(\mathrm{rms})$. Before ICP etching, substrates were cleaned using acetone and piranha. Etching experiments were conducted using ICP-98A inductively coupled plasma etching system developed by the Microelectronics R\&D center, Chinese Academy of Science. The etching parameters were: induction power $800 \mathrm{~W}$, substrate power $140 \mathrm{~W}$, bias voltage -220 $\mathrm{V}$, gas flow 50sccm $\mathrm{CHF}_{3}$, chamber pressure $1 \mathrm{~Pa}$. Surface morphologies after etching were scanned using a Dimension Edge atomic force microscope (AFM) from Bruker operating in tapping mode. The scan rate was $1 \mathrm{~Hz}$ and scan size was $10 \times 10 \mu \mathrm{m}^{2}$ with data resolution of 1024×1024. The tip used was HQ:NSC15/Al BS obtained from MikroMasch. After AFM test, morphological parameters, such as rms, height distribution function and dimensions of plasma induced nano-mounds were quantitatively obtained using standard analysis software 
(NanoScope Analysis, version 1.40) from Bruker unless otherwise noted. Etching depth was determined by Ambios XP-1 surface profiler.

\section{Results and discussions}

3.1. Nano-morphology transformation from dual-scale to one-scale

In figure 1, the atomic force microscopy (AFM) images and section profile of fused silica surface etched for 0.5 minute are shown. As we can see, submicron-size mounds develop on the etched surfaces. The mounds tend to be periodic and independent from each other. The areas between them are relatively smooth. This abrupt change of height indicates that the etched surface exhibits dual nano-scale morphology consisting of long wavelength underlying background surface and nano-mounds on top of it.

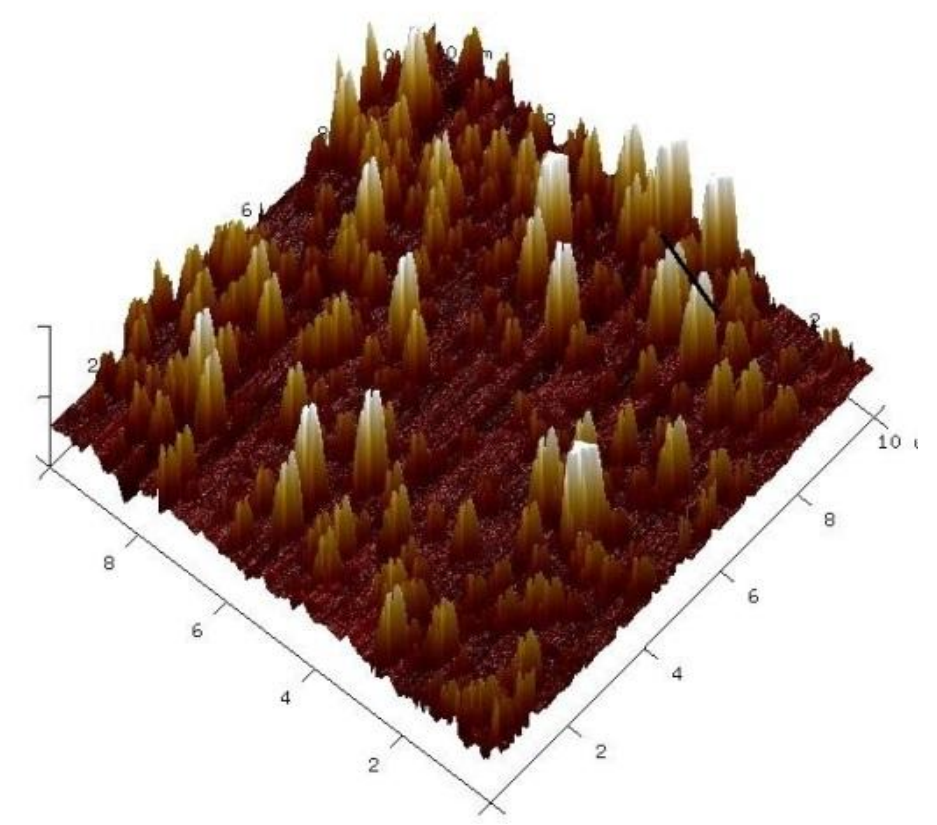

(a)

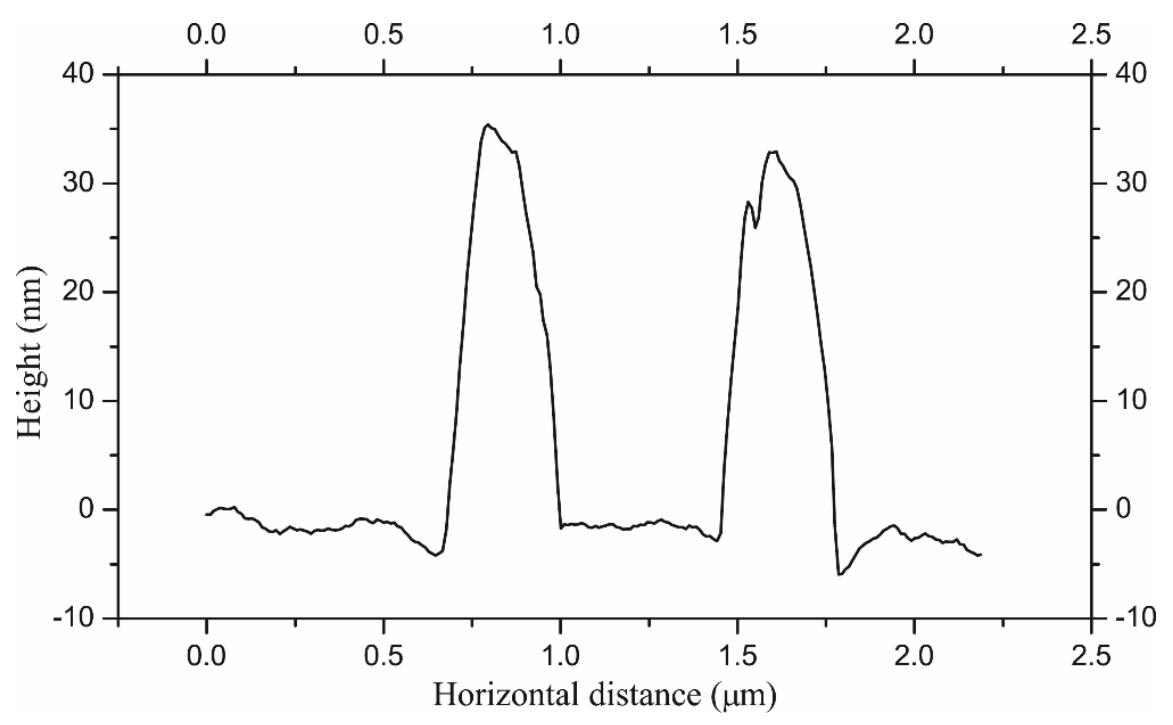

(b)

Fig.1. (a) 3D AFM image of fused silica surface etched for 0.5 minute. (b) Section profile of the AFM image shown in figure $1(\mathrm{a})$. 
Figure 2 shows the time evolution of surface morphology measured with AFM. As etching proceeds, the nano-mounds grow higher and wider. In the later period of etching, nano-mounds gradually coalesce with one another.

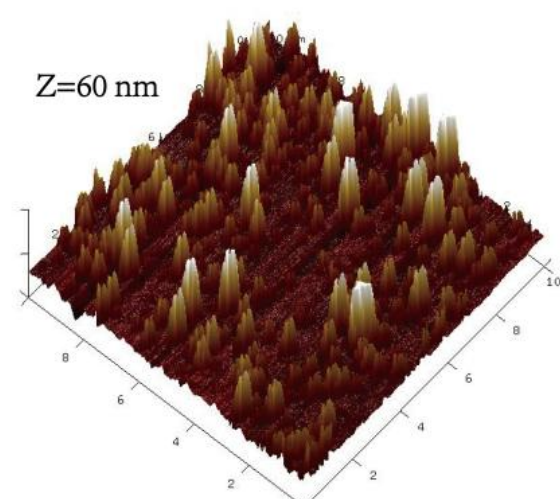

(a)

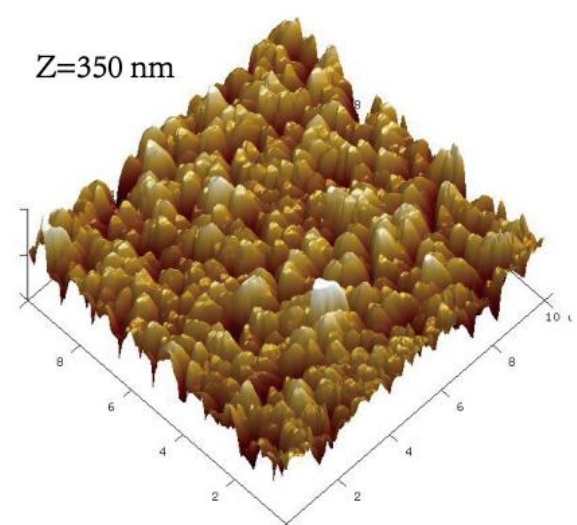

(d)

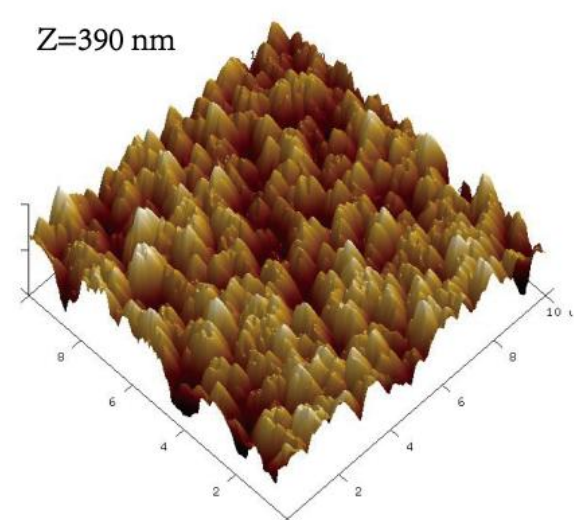

(g)

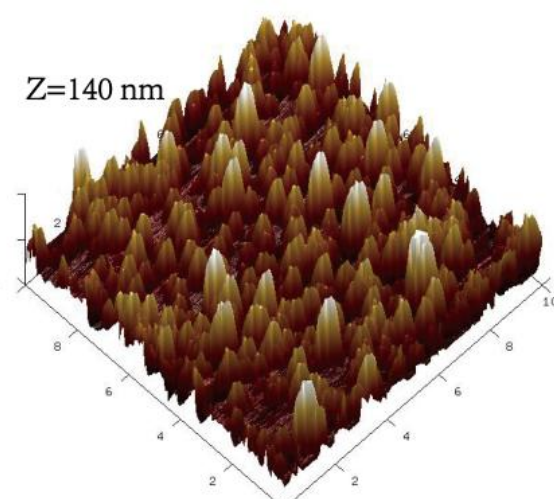

(b)

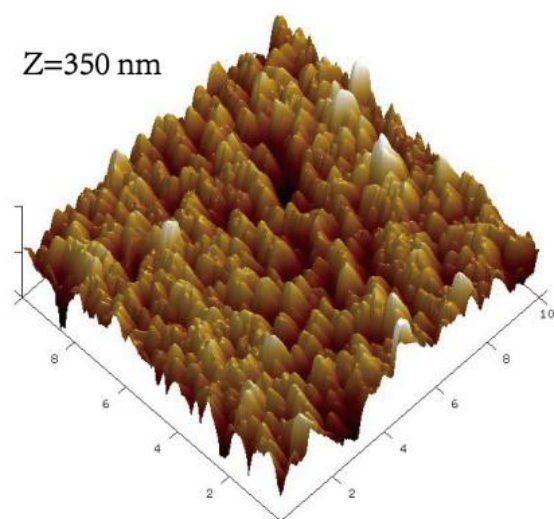

(e)

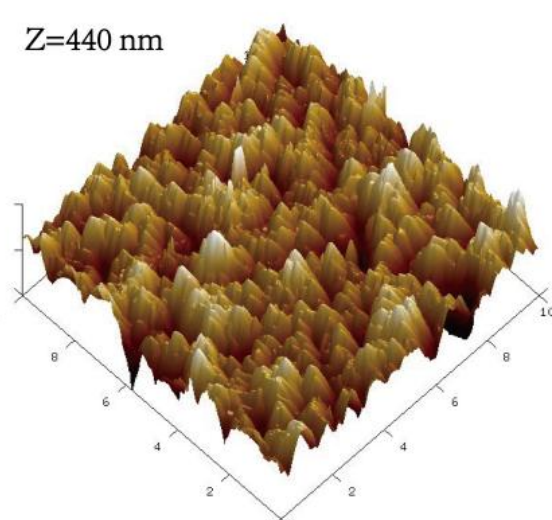

(h)

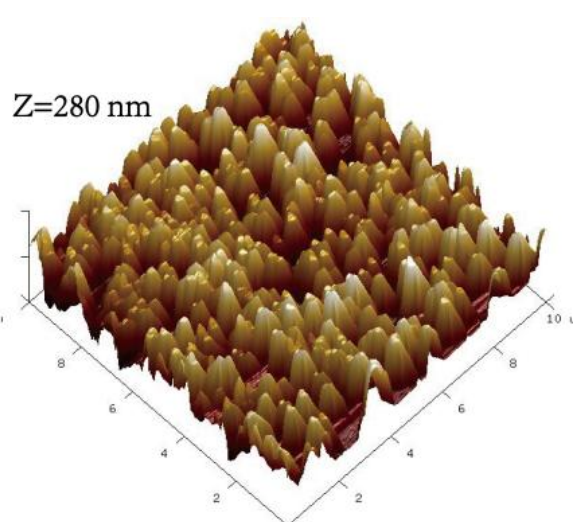

(c)

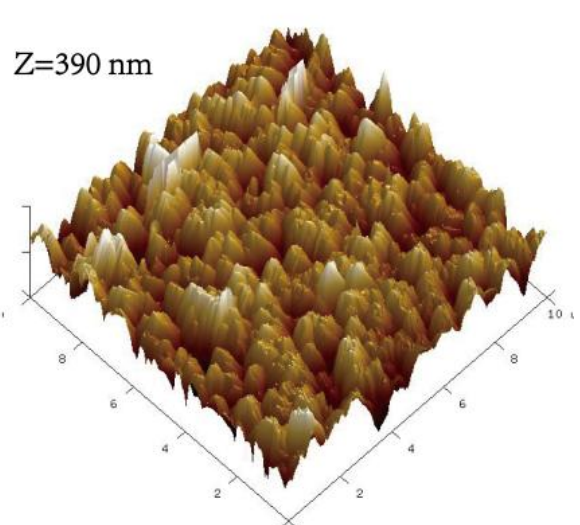

(f)

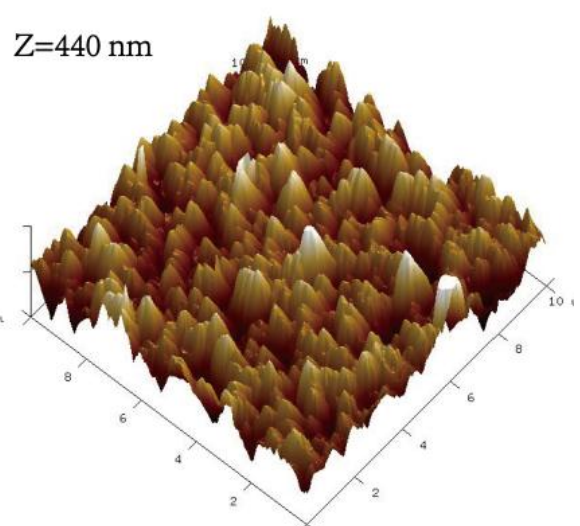

(i)

Fig.2. 3D AFM images of fused silica surface with different etching times: (a) $0.5 \mathrm{~min}$; (b) 1 min; (c) 2 min; (d) 4 min; (e) $6 \mathrm{~min}$; (f) $9 \mathrm{~min}$; (g) $12 \mathrm{~min}$; (h) $16 \mathrm{~min}$; (i) $30 \mathrm{~min}$. Z located at the top left corner of each subgraph presents the respective display range of $\mathrm{z}$ axis.

The dual-scale morphologies are also reflected in the height distribution functions plotted in figure 3. Before plasma etching, there is only one Gaussian peak, which is obviously due to the original roughness of polished surface. As etching begins, another Gaussian part representing the nano-mounds emerges, and grows with etching time. Specifically, for etching time longer than 2 minutes, the nano-mounds part becomes so dominant that the underlying 
roughness part vanishes, as shown in figure 6(e) and figure 6(f). This is confirmed by the section profile shown in figure 4 . As we can see, the nano-mounds have already coalesced, which indicates that the dual-scale morphology of nano-mounds and underlying surface has transformed into one-scale morphology of nano-mounds.

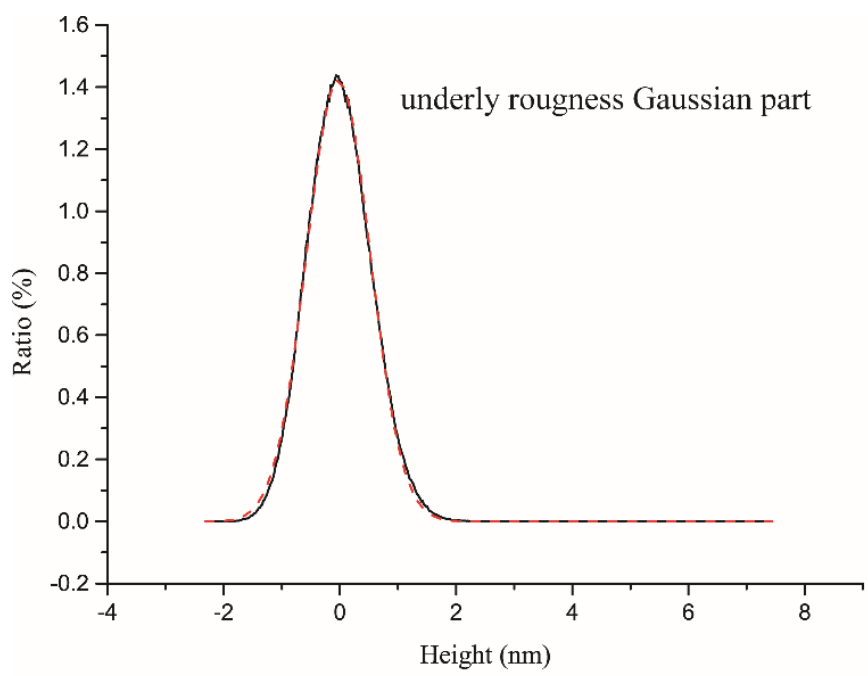

(a)

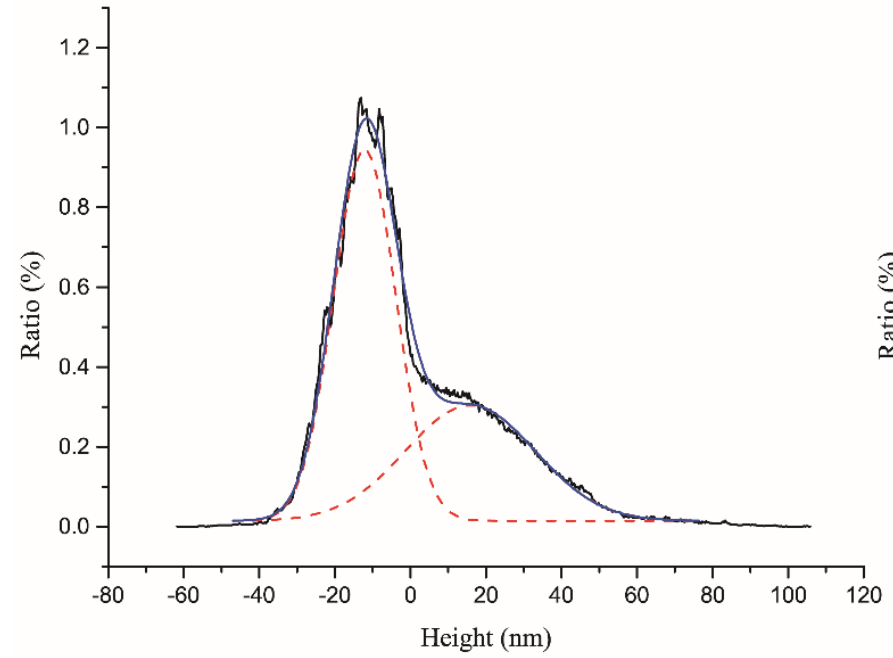

(c)

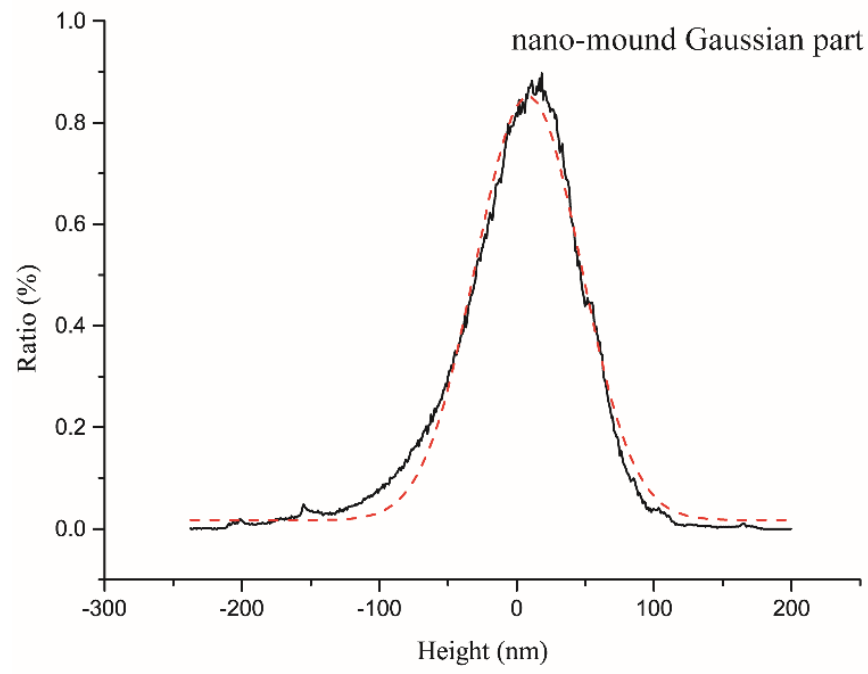

(e)

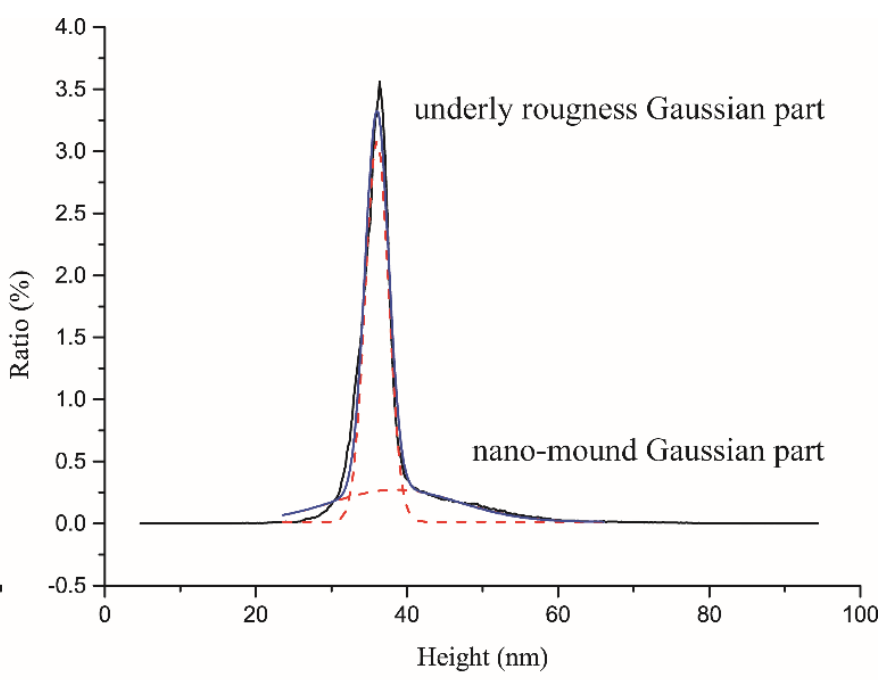

(b)

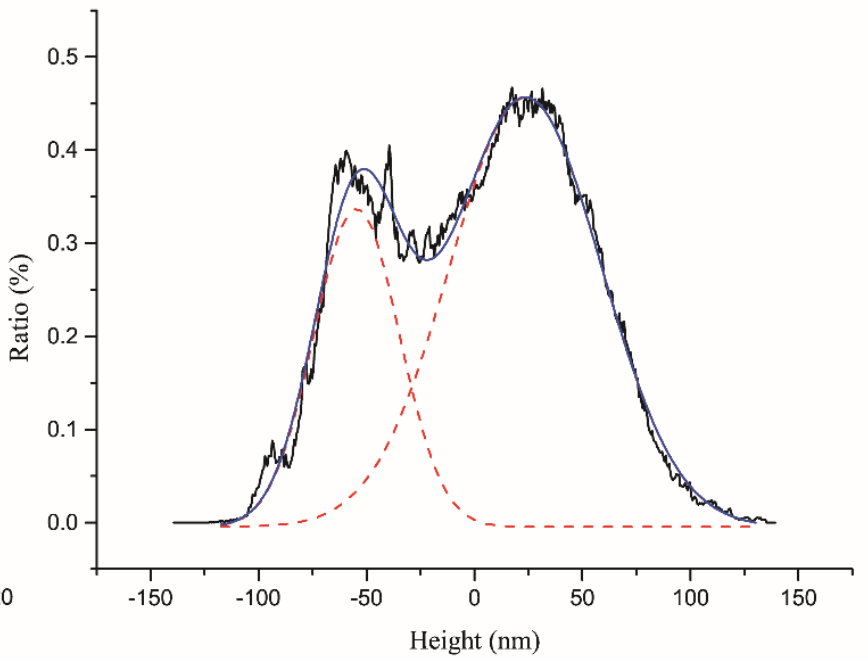

(d)

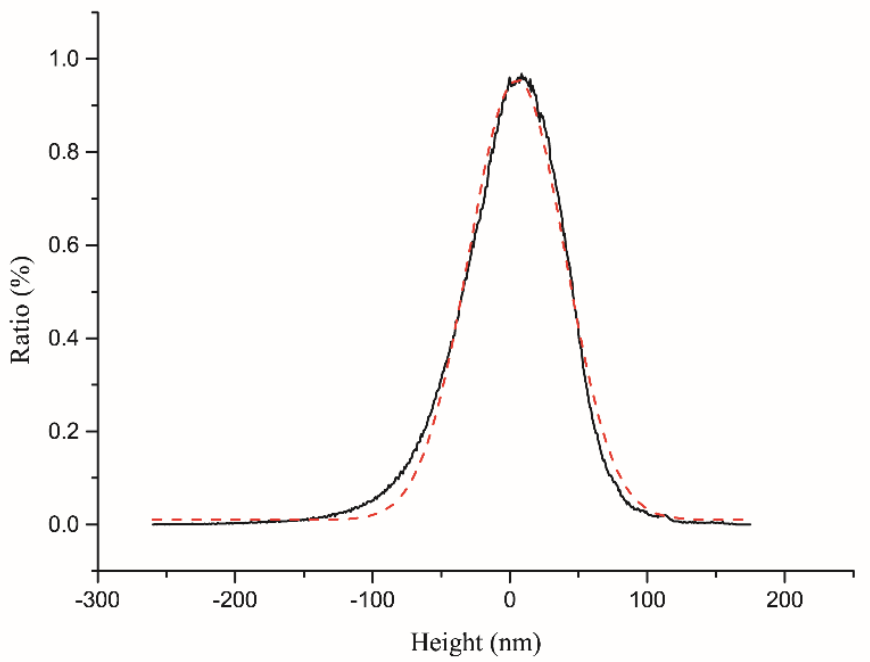

(f)

Fig.3. Plots of height distribution function for different etching times. (a) $0 \mathrm{~min}$; (b) $0.5 \mathrm{~min}$; (c) $1 \mathrm{~min}$; (d) $2 \mathrm{~min}$; (e) $4 \mathrm{~min}$; (f) 6 min. Etching time longer than 6 minutes exhibit exactly the same pattern as that of 4 and 6 minutes, therefore are not shown here because of the length of the picture. 


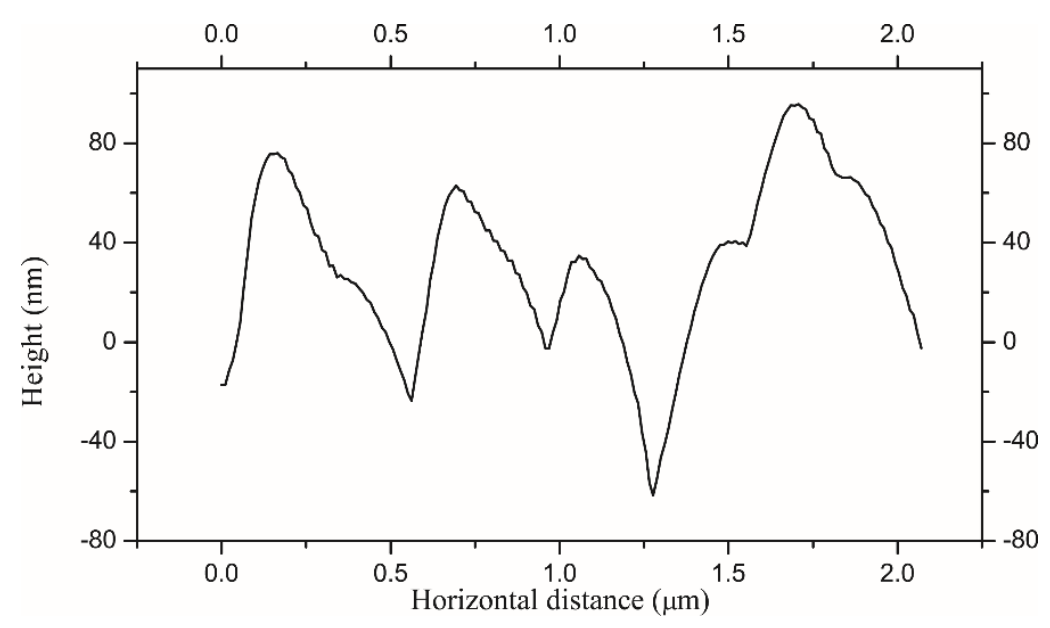

Fig.4. A random section profile of AFM image measured on fused silica surface etched for 4 minutes.

We also studied the evolution of etching rate verse etching time, see figure 5. Etching rate, i.e. the slope of the curve, dramatically decreases from $216 \mathrm{~nm} / \mathrm{min}$ for the first minute to 133 $\mathrm{nm} / \mathrm{min}$ after 30 minutes. This is probably due to the accumulation of etch inhibitors such as metal particles from the reactor chamber and less-volatile reaction products on etched surface, as will be detailed later. Such etching rate dependency on etching time has a negative effect on the further etching and hinders the prediction of etching depth.

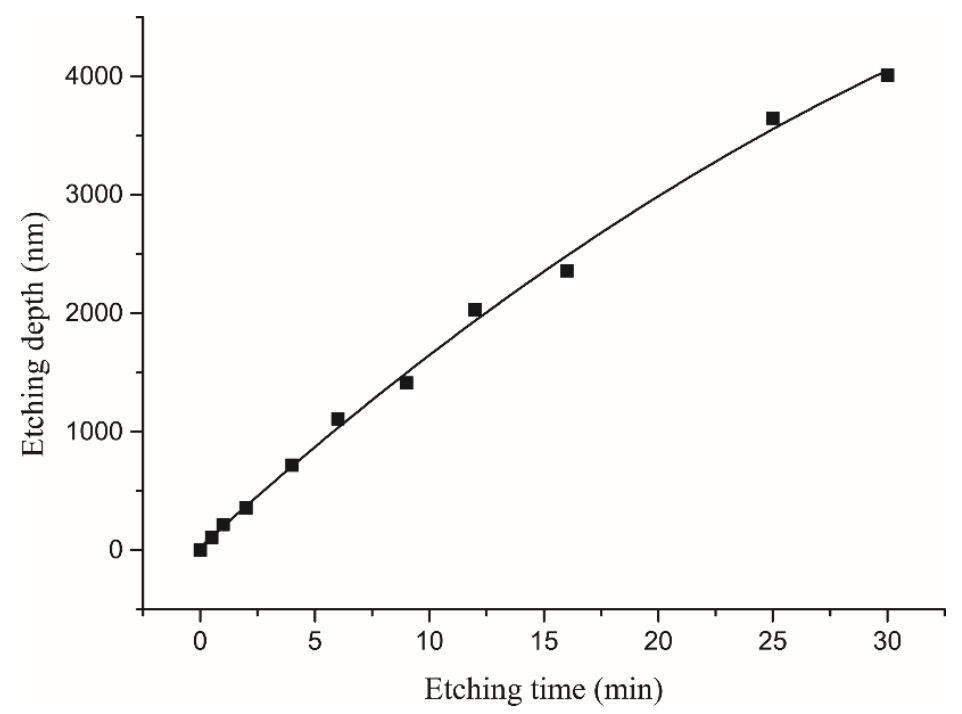

Fig.5. Etching depth versus etching time

\subsection{Bi-stage evolution of nano-morphology}

Figure 6(a) shows the time evolution of rms surface roughness. It is clearly seen that there are two evolution stages. At the beginning, rms increases linearly with etching time. During the second stage, the growth rate remains linear but drops to a much lower level. This differs from previous findings that rms increases linearly with etching time at a fixed rate. It is worth pointing that in previous works, researchers conventionally studied the morphological evolutions with respect to etching time instead of etching depth, assuming the etching process is time independent. However, considering the etching rate decreases with time in plasma roughening processes as previously discussed, we also studied rms evolution verse etching depth as shown in figure 6(b). And it exhibits the same pattern as time evolution. This excludes the possibility that the change of temporal evolution behaviors are caused by the etching rate dependency on etching time. In the later discussions on evolutions of other morphological parameters (correlation length and nano-mounds dimensions), the evolution behaviors of the two metrology methods all show the same trends so only time evolutions are presented. Figure 
7 shows the time evolution of correlation length. Similar to rms, correlation length also exhibits a bi-stage evolution. After a linear increase at the beginning, correlation length barely changes in the second stage.

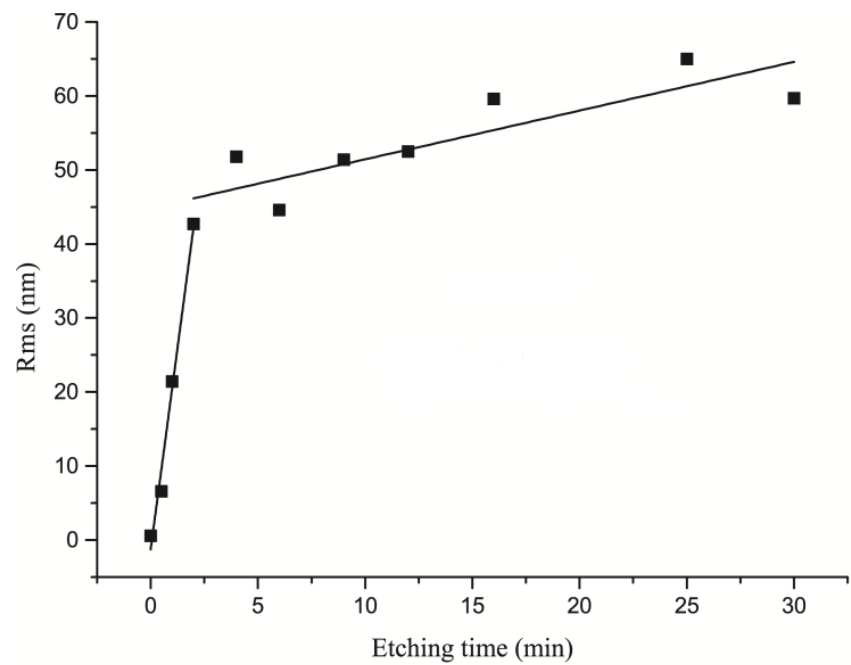

(a)

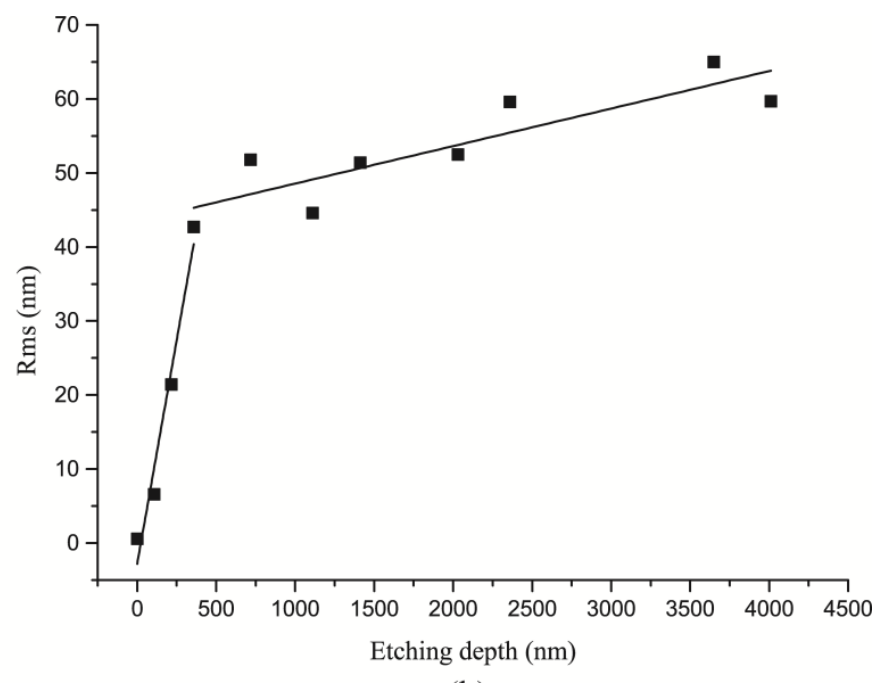

(b)

Fig.6. Rms of fused silica surfaces versus (a) etching time, (b) etching depth. The rms evolution verse etching depth exhibits almost the same pattern as that of etching time.

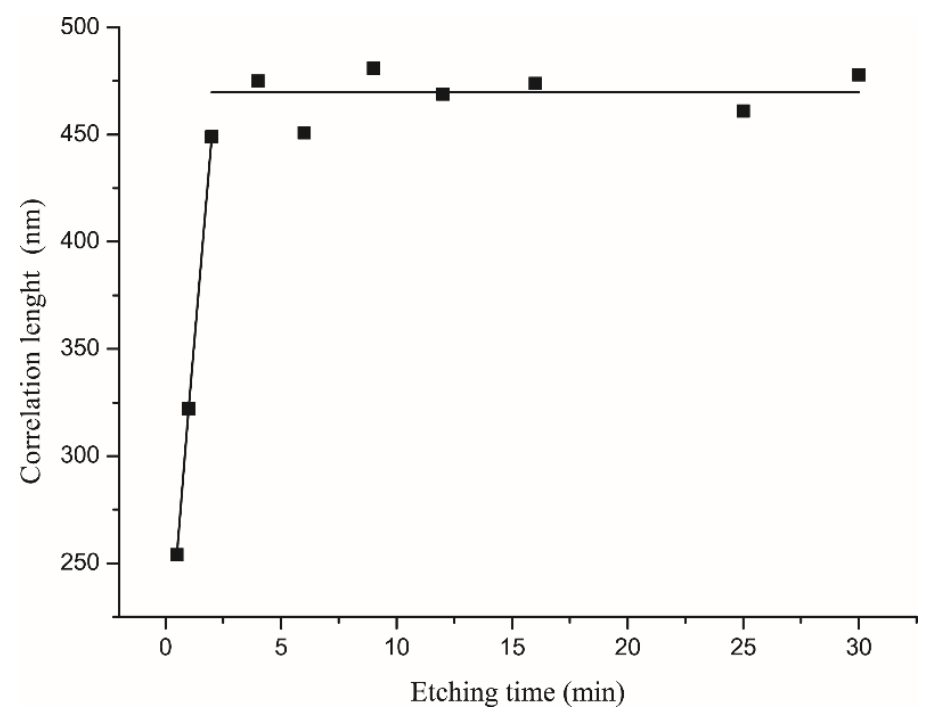

Fig.7. Correlation length versus etching time

Figure 8(a) shows the almost linear increase of nano-mound width verse etching time. This is consistent with our qualitative observation that the nano-mounds become wider as etching proceeds. For etching time longer than 2 minutes, the nano-mounds start to coalesce with one another so it is impossible to individually calculate diameter of the nano-mound. Then, based on the height distribution function plots shown in figure 3, time evolution of mound height is obtained. Height distribution accumulates height data within a specified area. Although it does not substitute for direct, cross-sectioning of the sample, it affords a means for comparing feature depth between two similar sites in a consistent, statistical manner. It is easy to understand that the horizontal range of height distribution plot reflects the height difference of the surface points. In our case, for instance, the width of the mound Gaussian peak (determined by the variance of Gaussian distribution) represents the height of mounds in a statistical way. Therefore, in order to quantitatively investigate the evolution of mound height, we define the full width at half maximum (FWHM) of mound Gaussian peak as the mean height of mounds. 
Thus, the time evolution of mean height is obtained, as shown in figure 8(b). At the beginning, mean height increases linearly at a rate of $41.47 \mathrm{~nm} / \mathrm{min}$. Then, the grow rate falls dramatically to $1.95 \mathrm{~nm} / \mathrm{min}$. Since $\mathrm{rms}$ roughness is mainly determined by the diameter and height of nanomound, this explains the sudden drop of growth rate of rms roughness.

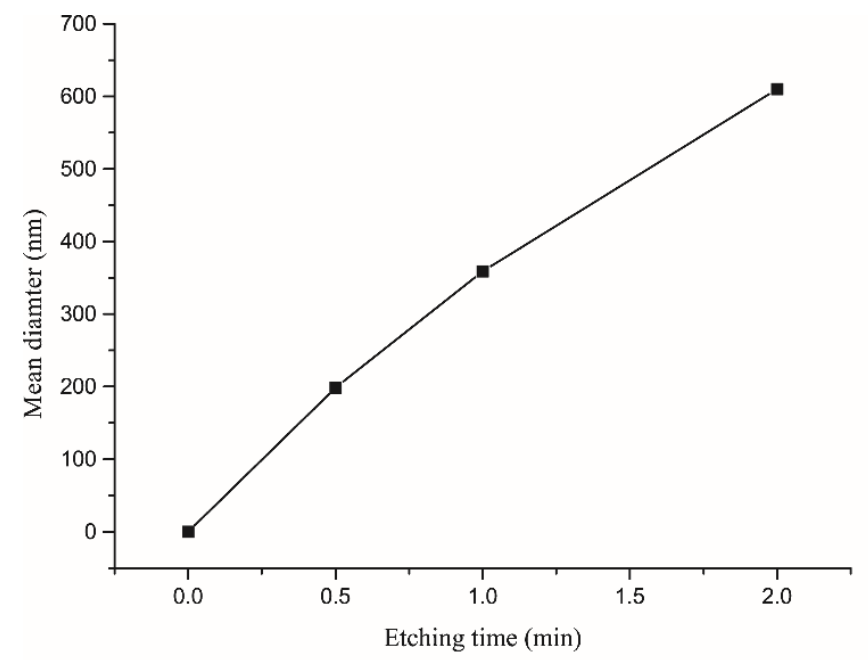

(a)

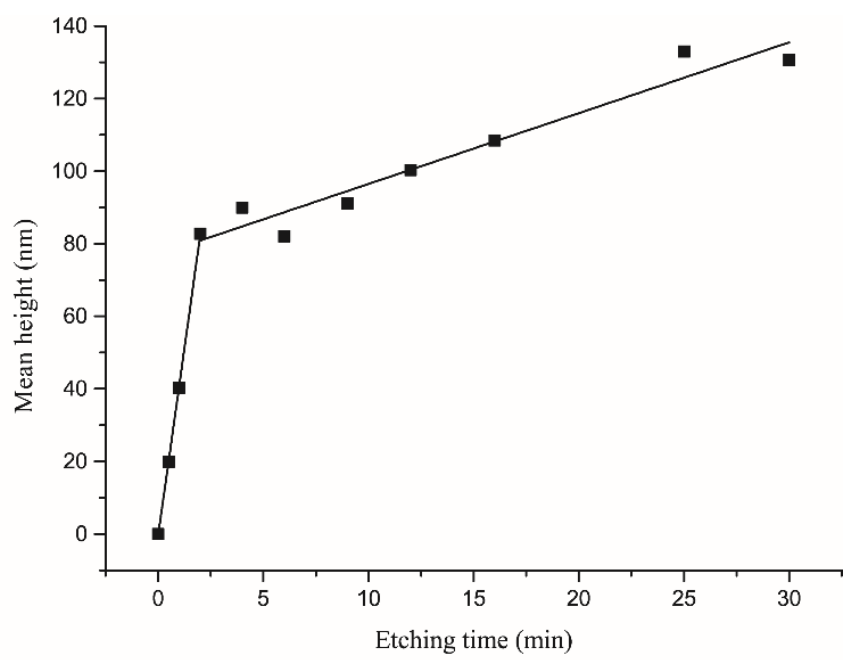

(b)

Fig.8. (a) Mean width, (b) Mean height of nano-mounds versus etching time.

The abrupt changes of morphological evolution behaviors observed in this work differ from any previous studies, and suggest a fundamental change of evolution mechanism. Moreover, the changes of evolution patterns synchronize with the morphology transformation from dual-scale to one-scale. This synchronism may be an important clue to the origin of the bi-stage evolution.

\subsection{Origin of the bi-stage evolution of nano-roughness}

Plasma induced surface roughening is generally attributed to the simultaneous deposition of non-volatile particles, i.e. etching inhibitors on etched surface during the etching process [6, 13, 14, 16-18]. Etching inhibitors are believed to induce roughness by causing local micromasking. In reference 17, G. Kokkoris et al proposed a model based on preferential deposition of etch inhibitors on the surface peaks to explain the roughness development observed in their $\mathrm{RIE}$ etching of $\mathrm{Si}$. There are generally four types of particles in a RIE system: ions, reactive neutrals, hard inhibitors and soft inhibitors. Hard inhibitors generally come from the metal parts of reactor chamber or hard mask, and can only be removed by ion. Soft inhibitors originate from the less-volatile CFx compounds or even reaction products, and can be removed by both ion and reactive neutrals. So they separately analyzed the depositions processes of hard and soft inhibitors with the assumption that the two processes are independent from each other. They argued that due to shadow effect, isotropic hard inhibitors would preferentially deposit on the surface peaks rather than valleys. The basic idea of shadow effect is that the crest of surface receives more deposited particles than the valley because the receiving solid angle at the peak is larger than that in the valley [22]. Since ion is anisotropic, all the etched surfaces receive the same ion flux. Thus the surface valleys will have a higher ratio of ion to hard inhibitor than peaks, which means a higher etch rate. As to soft inhibitor, in order to ensure the lower ratio of soft inhibitor to reactive neutrals on the surface peaks, they assume that soft inhibitors have a higher sticking probability than reactive neutral so that a smaller proportion of soft inhibitors will redistribute to the valleys compared to reactive neutrals. Given the fact that in high density plasma systems ion etching is dominant and reactive neutrals could be ignored, the second mechanism about soft inhibitor is not valid for our case. Moreover, this theory does not necessarily explain the dual-stage evolution observed in this work. 
In this work, we experimentally studied the respective effects of hard and soft etch inhibitors on nano-roughness by using a cup-shaped isolation device as shown in figure 9. By creating a semi-confined space, this device can effectively shield the substrate from the hard inhibitor sputtered from the chamber wall or substrate electrode. While, the substrate is still approachable for the soft inhibitor since it comes from the bulk plasma. More details about the isolation device can be found in our previous work [10].

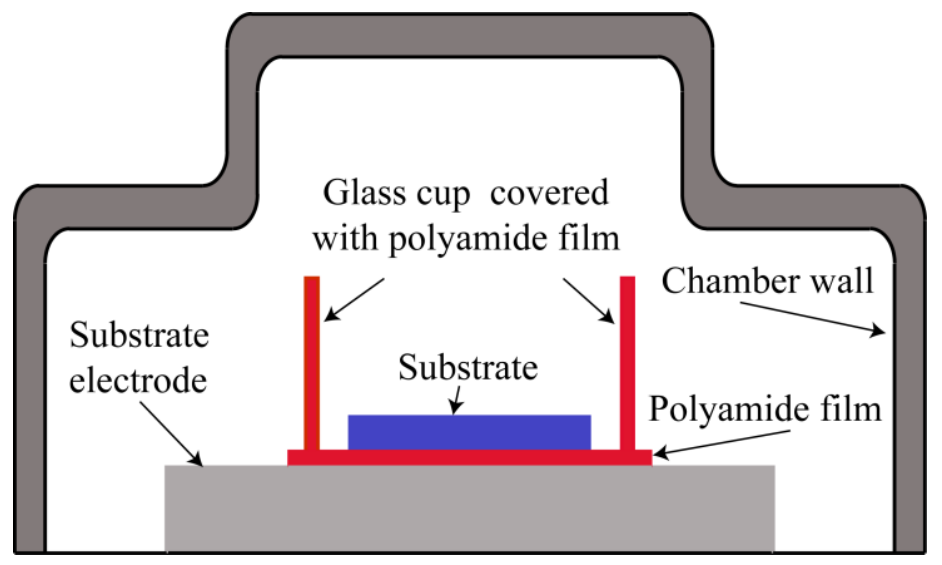

Fig.9. Schematic of isolation device

Table 1 shows the X-ray photoelectron spectroscopy (XPS) results measured on fused silica surfaces of different treatments: un-treated, conventionally etched, conventionally etched + piranha wet cleaning and isolated etched. For un-treated sample, there is no other element except the constituents of the fused silica. The small amount of $\mathrm{C}$ is due to the carbon contamination from air exposure [14]. After conventional ICP etching, aluminum, which is the main material of the reactor metal parts, is detected. Meanwhile, presence of fluorine and dramatic increase of carbon content imply the presence of soft inhibitor. Since our reactor is also used for etching SiN, the small amount of nitrogen probably comes from previous uses, which confirms that previous etching runs could also be influential. Then we try eliminating the etching inhibitors using Piranha wet cleaning. Piranha solution is a mixture of $\mathrm{H}_{2} \mathrm{SO}_{4} / \mathrm{H}_{2} \mathrm{O}_{2}$ and is very effective in removing polymer as well as metal contaminations [23]. However, after 1 hour of piranha $\left(\mathrm{H}_{2} \mathrm{SO}_{4}: \mathrm{H}_{2} \mathrm{O}_{2}=2: 1\right)$ cleaning, there are still noticeable amount of fluorine $(1.76 \%)$, carbon $(27.6 \%)$ and aluminum $(1.47 \%)$ left. This suggests that once residing on the etched surface, etch inhibitors seem to be very difficult to remove.

Table1. The composition of fused silica surface of different treatments obtained through high resolution XPS.

\begin{tabular}{|c|c|c|c|c|c|c|}
\hline \multirow[t]{2}{*}{ Treatments } & \multicolumn{6}{|c|}{ Elements (At. \%) } \\
\hline & $\mathrm{Si}$ & $\mathrm{O}$ & $\mathrm{F}$ & $\mathrm{C}$ & $\mathrm{Al}$ & $\mathrm{N}$ \\
\hline Un-treated & 24.82 & 68.96 & -- & 6.23 & -- & -- \\
\hline Conventional etched & 12.16 & 48.55 & 5.94 & 29.24 & 2.78 & 1.33 \\
\hline Con-etched + piranha & 16.51 & 52.66 & 1.76 & 27.6 & 1.47 & - \\
\hline Isolated etched & 24.28 & 65.77 & 2.5 & 7.45 & -- & -- \\
\hline
\end{tabular}

In isolated etching, however, aluminum is not detected. After hard inhibitor avoided, soft inhibitor is also greatly restrained: the contents of fluorine and carbon decrease from $5.94 \%$ and $29.24 \%$ to $2.5 \%$ and $7.45 \%$, respectively. This implies that hard inhibitor may play an important role in the attachment of soft inhibitor on the etched surface. This is reasonable because aluminum could act as a catalytic chain initiator for polymerization of $\mathrm{C}-\mathrm{F}$ radicals during plasma etching [14]. With the absence of hard inhibitor, plasma induced nano-roughness 
disappears, as shown in figure 10. Rms roughness decreases dramatically from $49.7 \mathrm{~nm}$ in conventional etching to $0.36 \mathrm{~nm}$ in isolated etching, which is even lower than the original roughness $(0.56 \mathrm{~nm})$. In conclusion, it is etch inhibitor that cause the formation of roughness. And the residence processes of hard and soft etch inhibitors are not independent from each other. Even though soft inhibitor participate the formation and development of roughness; it is hard inhibitor that plays the dominant role.

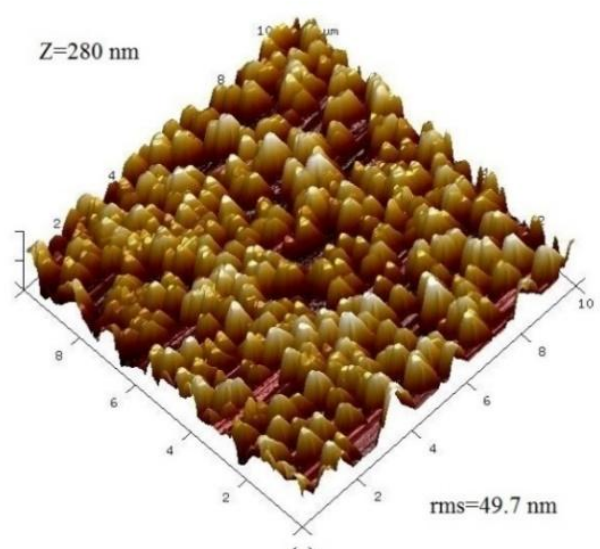

(a)

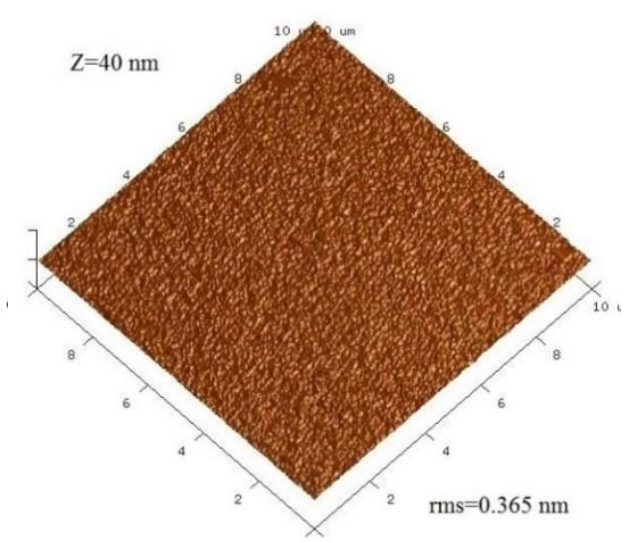

(b)

Fig.10. AFM images of fused silica surfaces etched for 2 minutes (a) conventionally etched; (b) isolated etched

In order to further explain the bi-stage morphological evolution, we proposed a simple model based on the topography transformation, see figure 11. At the beginning of etching, the mounds are small and discrete, so the surface morphology is dual-scale, as shown in figure 11(a). To make the model simpler, we assume that the mounds are covered with a layer of etch inhibitors while the underlying surface is naked fused silica. This is reasonable because the mound increase with etching time and the long wavelength underlying background surface is relatively smooth.

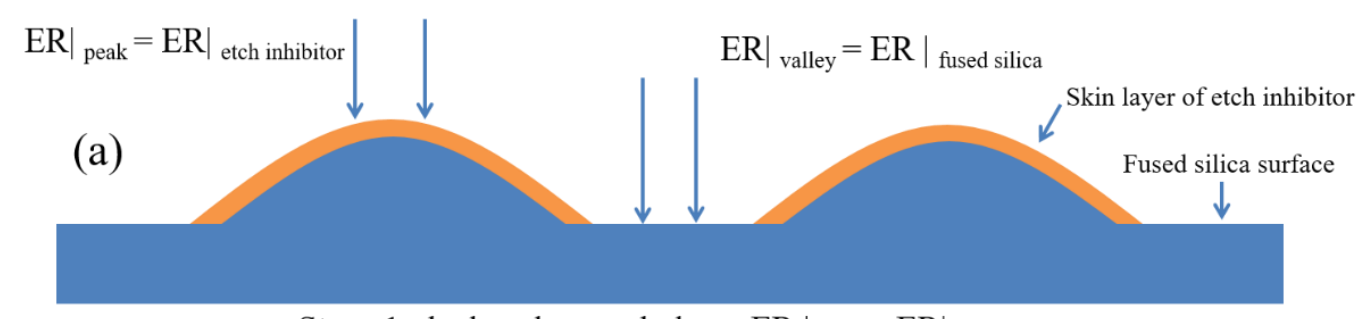

Stage 1: dual-scale morphology, ER $\left.\right|_{\text {peak }}<\left.\mathrm{ER}\right|_{\text {valley }}$

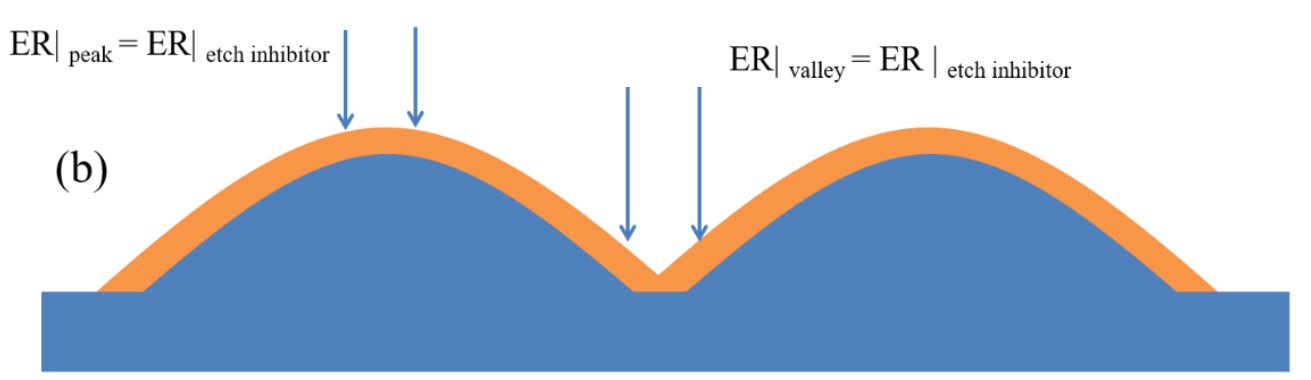

Stage 2: one-scale morphology, $\left.\mathrm{ER}\right|_{\text {peak }}=\left.\mathrm{ER}\right|_{\text {valley }}$

Fig.11. Mechanism for the bi-stage evolution of nano-morphology during the ICP etching of fused silica surface caused by difference of plasma etching resistance between surface peaks and valleys. ER is short for "etching rate". (a) Stage 1: dual-scale morphology; (b) Stage 2: one-scale morphology. 
As previously detailed, the main chemical compositions of etch inhibitor layer are aluminum compounds and $\mathrm{C}-\mathrm{F}$ polymer, which are both more resistant to $\mathrm{F}$-based plasma than fused silica. Therefore, there are actually two factors contribute to the inhomogeneity of local etch rate between the valley and peak: preferential deposition of etch inhibitor and difference of plasma etching resistance. This leads to the rapid linear increase of mean mound height as well as roughness in the first stage. As etching proceeds, the mounds grow laterally and after certain point coalesce with each other, as shown in figure 11(b). Consequently, the underlying background disappears and the surface topography transforms from dual-scale to one-scale. More importantly, the difference of plasma etching resistance between the peak and valley vanishes, resulting in the sudden drop of increase rate of mound height. This is also consistent with the fact that etching rate of stage 1 is much lower than that of stage 2 .

\section{Summary}

We experimentally investigated the development of nano-roughness during the ICP etching of fused silica, and discover a novel bi-stage time evolution of nano-morphology. At the beginning of etching, the etched surface roughens rapidly with increase of etching time. At the second stage, roughening process slows down remarkably. Moreover, the switching of evolution stages synchronizes with the morphological transformation from dual-scale of nano-mounds and underlying background surface to one-scale of nano-mounds. Then we explored the origin of the nano-roughness by separately studying the effects of hard and soft inhibitor using a cupshaped isolation device, and found that it is hard inhibitor that plays a dominant role in the surface roughening. At last, a theoretical model based on morphology transformation was proposed. The key idea is that for dual-scale morphology, both preferential deposition of etch inhibitors and difference of plasma etching resistance between surface valleys and peaks contribute to the roughness development. After morphology transforming into one-scale, difference of plasma etching resistance vanishes, thus the roughening process slows down. This theory agrees with the fact that etching rate of stage 1 is much lower than that of stage 2 .

\section{Acknowledge}

The research was supported by the Major Program of the National Science Foundation of China (Grant No. 51535003 and grant no. 10975139), the Young Scientists Fund of the National Natural Science Foundation of China (Grant No. 11404301 and 61505185), the Knowledge Innovation Program of the Chinese Academy of Sciences (grant no. GY2011053005), and the National Major Project of China.

\section{References}

[1] J..H. Campbell, R.A. Hawley-Fedder, C.J. Stolz, J.A. Menapace, M.R. Borden, P.K. Whitman, J. Yu, M. Runkel, M.O. Riley, M.D. Feit, and R.P. Hackel, NIF optical materials and fabrication technologies: an overview, Proc. of SPIE 5341, (2004) 84-95.

[2] J..A. Menapace, B. Penetrante, D. Golini, A. Slomba, P.E. Miller, T. Parham, M. Nichols, and J. Peterson, Combined advanced finishing and UV-laser conditioning for producing UV-damage-resistant fused silica optics, Proc. of SPIE 4679, (2002) 56-66.

[3] S. Q. Wang, C. H. Zhou, H. Y. Ru, and Y. Y. Zhang, Optimized condition for etching fused-silica phase gratings with inductively coupled plasma technology, Appl. Opt. 44, (2005) 4429-4434.

[4] C.C. Zhang, G. Hatipoglu, S. Tadigadapa, High speed, ultra smooth etching of fused silica substrate in $\mathrm{SF}_{6}, \mathrm{NF}_{3}$ and $\mathrm{H}_{2} \mathrm{O}$ based inductively coupled plasma process, J. Microelectromech. Syst. 24 (2014) 922-930.

[5] H. .Jin, Q. Xin, N. Li, J. Jin, B. Wang, and Y. Yao, The morphology and chemistry evolution of fused silica surface after Ar/CF 4 atmospheric pressure plasma processing, Appl. Surf. Sci. 286 (2013) 405-411. 
[6] L. X. Sun, H. J. Liu, J. Huang, X. Ye, Q. Cheng, X. D. Zhou, F. R. Wang, X. D. Jiang, and W. D. Wu, The effect of RIE-modified surface contamination on optical, Proc. SPIE 8786, (2013) 87860P.

[7] L. E. Busse, C.M. Florea. J. A. Frantz, Anti-reflective surface structures for spinel ceramics and fused silica windows, lenses and optical fibers, Thin. Solid Film 519 (2014) 3792-3797.

[8] P. Sheng, S. M. Xiong, Q. L. Deng, Disordered antireflective subwavelength structures using Ag nanoparticles on fused silica window, Appl. Opt. 53 (2014) 6789-6796.

[9] Y. P. Zhao, Jason T. Drotar, G.-C. Wang, and T.-M. Lu, Roughening in Plasma Etch Fronts of Si (100), Phys. Rev. Lett. 82, (1999) 4882-4885.

[10] Xiaolong Jiang, Ying Liu, Zhengkun Liu, Optimum inductively coupled plasma etching of fused silica to remove subsurface damage layer, Appl. Surf. Sci. 355 (2015) 1180-1185.

[11] J. Zhou, Y. Chen, W. Zhou, X. Miao, ZheYang, N. Yu, H. Liu, T. Lan, and J. Yan, "Inductively coupled plasma etching for phase-change material with superlattice-like structure in phase change memory device," Appl. .Surf. Sci. 280, (2013) 862-867.

[12] A. Goyal, V. Hood, and S. Tadigadapa, High speed anisotropic etching of Pyrex ${ }^{\circledR}$ for microsystems applications, J. Non-Cryst. Solids. 352 (2006) 657-663.

[13] E. Gogolides, V. Constantoudis, G. Kokkoris, D. Kontziampasis, K. Tsougeni, G. Boulousis, M. Vlachopoulou, and A. Tserepi, Controlling roughness: from etching to nanotexturing and plasma-directed organization on organic and inorganic materials, J.Phys D-Appl.Phys. 44 (2011) 174021.

[14] D. Y. Choi, J. H. Lee, D. S. Kim, and S. T. Jung, Formation of plasma induced surface damage in silica glass etching for optical waveguides, J. Appl. Phys. 95 (2004) 8400-8407.

[15] S. H. Lee and J.-Q. Lu, Nano-ring-shape growth of fluorocarbon macromolecules during $\mathrm{SiO}_{2}$ etching, Nanotechnology 21(2010) 155303.

[16] L. Li, T. Abe, and M. Esashi, Smooth surface glass etching by deep reactive ion etching with $\mathrm{SF}_{6}$ nd Xe gases, J. Vac. Sci \& Technol B: Microelectron. Nano. Structs. 21 (2003) 2545-2550.

[17] G. Kokkoris, V. Constantoudis, P. Angelikopoulos, Dual nanoscale roughness on plasma-etched Si surfaces: Role of etch inhibitors, Phys. Rev. B 76 (2007) 193405.

[18] G. Boulousis, V. Constantoudis, G. Kokkoris, Formation and metrology of dual scale nano-morphology on SF6 plasma etched silicon surfaces, Nanotechnology 19 (2008) 255301.

[19] N. Nakazaki, H. Tsuda, Y. Takao, Two modes of surface roughening during plasma etching of silicon: Role of ionized etch products, J.Appl.Phys. 116 (2014) 223302.

[20] J. Bae and I. J. Lee, A bifractal nature of reticular patterns induced by oxygen plasma on polymer films. Sci. Rep. 5 (2015) 10126.

[21] Maryam Alsadat Rad, Kamarulazizi Ibrahim and Khairudin Mohamed, Formation of $\mathrm{SiO}_{2}$ surface textures via $\mathrm{CHF}_{3} / \mathrm{Ar}$ plasma etching process of poly methyl methacrylate self-formed masks, Vacuum, 101 (2014) 67-70.

[22] M. Martin and G. Cunge, Surface roughness generated by plasma etching processes of silicon, J. Vac. Sci. Technol. B, 26(2008) 1281-1288.

[23] W. Kern, Handbook of Semiconductor Wafer Cleaning Technology: Science, Technology, and Applications,Materials Science and Process Technology Series, pp. 3-56, Noyes Publications, Park Ridge, NJ, 1993. 